\title{
NOVOS MATERIAIS À BASE DE NANOFIBRAS DE CARBONO COMO SUPORTE DE CATALISADOR NA DECOMPOSIÇÃO DA HIDRAZINA
}

\author{
Ricardo Vieira ${ }^{* \#}$ \\ Instituto Nacional de Pesquisas Espaciais, Rodovia Presidente Dutra, Km 40, 12630-000 Cachoeira Paulista - SP \\ Cuong Pham-Huu, Nicolas Keller e Marc J. Ledoux
}

Laboratoire des Matériaux, Surface et Procédés pour la Catalyse, Université Louis Pasteur, 25 rue Becquerel, 67087 Strasbourg

Cedex 2, France

Recebido em 25/7/02; aceito em 22/1/03

\begin{abstract}
CARBON NANOFIBERS A NEW CATALYST SUPPORT FOR HYDRAZINE DECOMPOSITION. Today satellites propulsion is based on the use of monopropellant and/or bipropellant chemical systems. The maneuvering of satellite is based on the hydrazine decomposition micropropulsors catalyzed by metallic iridium supported on $\gamma$-alumina. This reaction is a surface reaction and is strongly exothermic and implies that the operation of the micropropulsor is controlled by the mass and heat diffusions. For this reason and for the fact that the propulsor operation is frequently in pulsed regime, the catalyst should support high pressure and temperature variations within a short time period. The performance and the durability of the commercial catalyst are jeopardized by the low thermal conductivity of the alumina. The low thermal conductivity of the alumina support restricts the heat diffusion and leads to the formation of hot spots on the catalyst surface causing the metal sintering and/or fractures of the support, resulting in loss of the activity and catalyst destruction. This work presents the synthesis and characterization of new carbon composite support for the active element iridium, in substitution of the commercial catalysts alumina based support. These supports are constituted of carbon nanofibers (30 to $40 \mathrm{~nm}$ diameter) supported on a macroscopic carbon felt. These materials present high thermal conductivity and mechanical resistance, as well as the easiness to be shaped with different macroscopic shapes. The mechanical stability and the performance of the iridium supported on the carbon composite support, evaluated in a laboratory scale test in hydrazine decomposition reaction, are superior compared to the commercial catalyst.
\end{abstract}

Keywords: carbon nanofiber; hydrazine; catalytic decomposition.

\section{INTRODUÇÃO}

Há satélites de telecomunicação que são estabilizados por rotação em torno de um único eixo, co-linear ao seu vetor velocidade. As antenas são colocadas sobre uma plataforma rotativa no sentido oposto a sua rotação de estabilização e direcionadas à terra. Os painéis solares são dispostos em torno do satélite. A propulsão da maior parte dos satélites atuais em funcionamento é efetuada por sistemas químicos a monopropelentes (hidrazina) ou a bipropelentes (monometil hidrazina e tetróxido de nitrogênio), ou ainda, por uma combinação destes sistemas.

O sistema propulsivo mais empregado é aquele que utiliza a decomposição catalítica de hidrazina. Este sistema é constituído de um reservatório de monopropelente, de uma linha de alimentação, de uma eletroválvula e de micropropulsores. O micropropulsor, esquematizado na Figura 1, é composto basicamente de um sistema de injeção, de um leito catalítico e de uma tubeira. O injetor tem por objetivo aspergir o propelente e otimizar a sua distribuição sobre o leito catalítico. O leito catalítico pode ser dividido, de maneira geral, em duas regiões. Na primeira, mais próxima do injetor a hidrazina é vaporizada e decomposta cataliticamente. Na região seguinte, o restante da hidrazina e a amônia produzida anteriormente são decompostas termicamente e/ou cataliticamente em hidrogênio e nitrogênio.

*e-mail: vieirar@ecpm.u-strasbg.fr

\# endereço atual: Laboratoire des Matériaux, Surface et Procédés pour la Catalyse, 25 rue Becquerel, 67087 Strasbourg Cedex 2, France

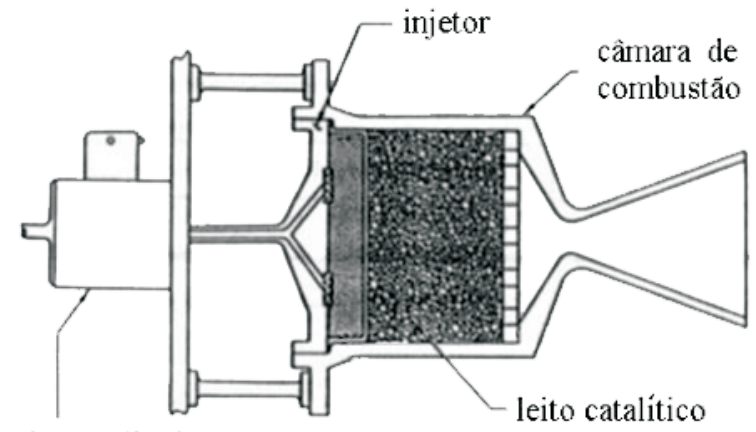

eletro-válvula

Figura 1. Esquema de um micropropulsor a decomposição catalítica de hidrazina

Dependendo das condições de funcionamento do propulsor e do catalisador utilizado, a hidrazina pode decompor-se, basicamente, segundo as reações ${ }^{1}$ :

$3 \mathrm{~N}_{2} \mathrm{H}_{4} \rightarrow \mathrm{N}_{2}+4 \mathrm{NH}_{3}$ $3 \mathrm{~N}_{2} \mathrm{H}_{4} \rightarrow 3 \mathrm{~N}_{2}+6 \mathrm{H}_{2}$

A reação de decomposição do monopropelente hidrazina é exotérmica e deve ocorrer o mais rápido possível para o bom funcionamento do micropropulsor ${ }^{2}$. Portanto a liberação da massa e a dissipação do calor gerados durante a reação devem também ocorrer rapidamente, pois em alguns milisegundos há um aumento drástico 
da temperatura e da pressão no leito catalítico, podendo atingir $1000{ }^{\circ} \mathrm{C}$ e 20 bar, respectivamente. Torna-se então necessário a utilização de um catalisador com uma macroporosidade que permita um rápido escoamento dos gases formados, com uma excelente resistência mecânica e uma grande interação metal-suporte que evite a perda da fase ativa ${ }^{3}$. É também desejável o emprego de suportes que apresentem uma condutividade térmica suficiente para evitar a formação de pontos superaquecidos na superfície do catalisador e, conseqüentemente, a sinterização da fase metálica.

Atualmente, todos os sistemas de micropropulsão de satélites a decomposição da hidrazina utilizam como catalisador o irídio suportado em alumina contendo de 30 a $40 \%$ em massa do metal. Apesar dos progressos apresentados na obtenção de suportes mais estáveis, o catalisador comercial ainda apresenta algumas limitações, tais como baixa condutividade térmica e uma superfície específica gerada por uma rede de poros. A baixa condutividade tende à formação indesejável de pontos superaquecidos, enquanto a rede de poros não facilita o escoamento dos gases gerados pela reação.

Recentemente tem sido sugerida a aplicação de nanotubos e nanofibras de carbono como adsorventes, como suportes de catalisadores $^{4}$ e como armazenadores de hidrogênio ${ }^{5}$. Estes materiais podem ser produzidos de diferentes maneiras, tais como descarga de arco elétrico ${ }^{6}$, vaporização por laser ${ }^{7}$ e por decomposição catalítica $^{8}$. Dentre estes métodos, o catalítico é o mais simples, o mais barato e aquele que produz a menor quantidade de carbono amorfo paralelamente aos nanotubos, não necessitando assim de uma posterior purificação. Este método consiste na decomposição catalítica ( $\mathrm{Fe}, \mathrm{Ni}$ e $\mathrm{Co}$ ) de alguns hidrocarbonetos a temperaturas que variam de 400 a $800{ }^{\circ} \mathrm{C}$. O método catalítico pode ser empregado tanto na produção de nanofibras como na de nanotubos de carbono. A distinção entre estes dois materiais não é muito clara. Os nanotubos de carbono são constituídos por folhas de grafite enroladas e paralelas ao eixo dos tubos, apresentando na superfície os planos basais poucos reativos. As nanofibras de carbono são constituídas por placas de grafite empilhadas ao longo do eixo da fibra na forma de cone, apresentando os planos prismáticos expostos e bastante reativos.

Uma das principais características destas estruturas é a presença de um grande número de extremidades que favorecem as interações físicas ou químicas, particularmente a adsorção. O mais surpreendente é que tais sólidos, compostos de cristais ordenados, podem apresentar altas áreas específicas $\left(100-500 \mathrm{~m}^{2} / \mathrm{g}\right)$, onde a totalidade de sua superfície é quimicamente ativa. Estes materiais apresentam também boa resistência mecânica e alta condutividade térmica ${ }^{9}$. Do ponto de vista físico, estes materiais podem variar de 5 a $100 \mathrm{~nm}$ de diâmetro e de 5 a $100 \mu \mathrm{m}$ de comprimento.

A utilização dos nanotubos e nanofibras de carbono em reatores de configuração clássica ainda é limitada. $\mathrm{O}$ tamanho nanométrico do substrato dificulta o seu emprego em leito fixo, devido a problemas de perda de carga causada pelo alto grau de empacotamento e a problemas de arraste do catalisador pelo gás reacional. O emprego mais eficaz destes materiais poderá ser viabilizado apenas com a preparação de nanofibras e nanotubos de carbono na forma macroscópica ${ }^{10}$.

Este trabalho tem o objetivo de apresentar a síntese de nanofibras de carbono sobre uma superfície macroscópica, bem como sua aplicação como suporte do catalisador de irídio na reação de decomposição catalítica da hidrazina para fins aeroespaciais. Os desempenhos deste novo material nesta reação serão comparados aos do catalisador comercial $\left(36 \% \mathrm{Ir} / \mathrm{Al}_{2} \mathrm{O}_{3}\right)$.

\section{PARTE EXPERIMENTAL}

Como material precursor foi utilizado feltro de carbono $\left(1 \mathrm{~m}^{2} / \mathrm{g}\right)$ fornecido pela Carbone Lorraine Co., composto de microfibras de carbono entrelaçadas medindo $10 \mu \mathrm{m}$ de diâmetro e alguns milímetros de comprimento. Este material foi cortado na forma de pastilhas cilíndricas medindo $1 \mathrm{~cm}$ de diâmetro e $0,5 \mathrm{~cm}$ de altura. As pastilhas foram impregnadas com $1 \%$ em peso do metal níquel, utilizando-se uma solução alcoólica (20\% etanol) de nitrato de níquel (Merck). O material foi deixado a temperatura ambiente por $12 \mathrm{~h}$ seguido de etapas de secagem a $100{ }^{\circ} \mathrm{C}$ e calcinação a $350{ }^{\circ} \mathrm{C}$, ambas por $2 \mathrm{~h}$. Após $1 \mathrm{~h}$ de redução a $400{ }^{\circ} \mathrm{C}$, o hidrogênio (Air liquide) foi substituído por uma mistura reacional contendo hidrogênio e etano (Air liquide), empregando uma razão volumétrica de 5:1 $\left(\mathrm{H}_{2}: \mathrm{C}_{2} \mathrm{H}_{6}\right)$. $\mathrm{O}$ precursor foi mantido sob esta mistura a $700{ }^{\circ} \mathrm{C}$ durante $2 \mathrm{~h}$ e, nestas condições, ocorreu a síntese das nanofibras de carbono na superfície do feltro.

As pastilhas contendo nanofibras de carbono foram impregnadas com $30 \%$ em peso do elemento ativo irídio, utilizando-se para este fim o ácido $\mathrm{H}_{2} \mathrm{IrCl}_{6}$ (Acros) em solução alcoólica (80\% etanol). Este material foi deixado à temperatura ambiente por $12 \mathrm{~h}$, posteriormente secado a $100{ }^{\circ} \mathrm{C}$, calcinado a $350{ }^{\circ} \mathrm{C}$ e reduzido a $400{ }^{\circ} \mathrm{C}$, todas estas etapas por $2 \mathrm{~h}$.

Os catalisadores assim preparados foram testados em um sistema de ensaios que consiste em micro injeções do propelente sobre um leito catalítico confinado (Figura 2). O sistema empregado visa simular as condições de operação de um micropropulsor de satélites e tem como objetivo medir o tempo de ignição da reação, ou seja, o tempo compreendido entre o acionamento da eletroválvula e o valor de pressão gerado na câmara de reação. Para isto utilizou-se micro injeções $(0,5 \mathrm{~s})$, aspergindo $0,34 \mathrm{ml}$ de hidrazina, pressurizada a 2 bar, sobre $100 \mathrm{mg}$ de catalisador confinado a pressão ambiente. A pressão na câmara de reação foi monitorada por um medidor de pressão da marca Keller, modelo PA-23S, obtendo-se 10 medidas por segundo. $\mathrm{O}$ desempenho do catalisador à base de nanofibras foi comparado ao do catalisador comercial, submetido às mesmas condições.

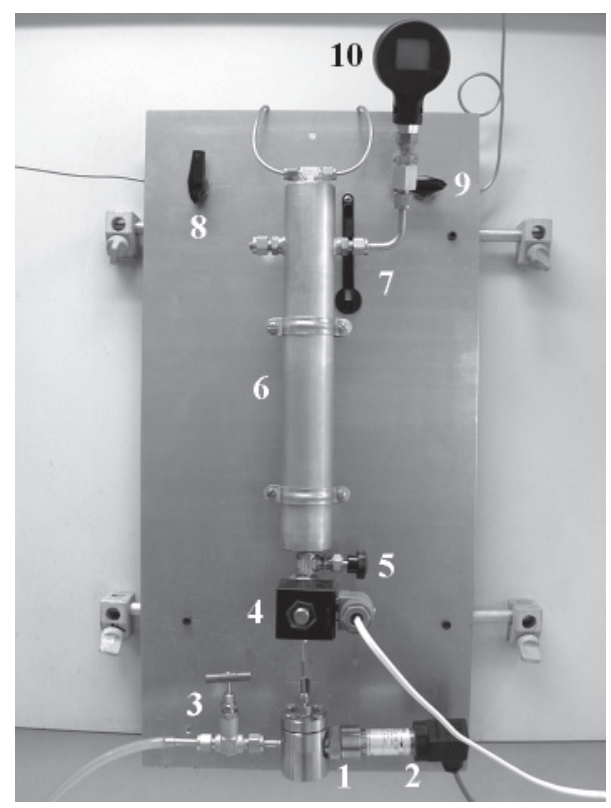

Figura 2. Fotografia do sistema de ensaios de ignição da reação de decomposição da hidrazina: 1. câmara de reação, 2. medidor de pressão, 3. válvula de descarga, 4. eletroválvula, 5. válvula de segurança, 6 . reservatório de hidrazina, 7. detetor de gases tóxicos, 8. válvula de pressurização, 9. válvula de alivio e 10. manômetro 


\section{Caracterização}

A morfologia dos catalisadores foi observada em um Microscópio Eletrônico de Varredura (MEV), marca Jeol e modelo JSM-840, trabalhando a $40 \mathrm{kV}$ e $20 \mathrm{~mA}$. As características microestruturais das nanofibras de carbono, bem como a dispersão das partículas nanométricas do metal irídio sobre a superfície do suporte foram observadas em um Microscópio Eletrônico de Transmissão (MET) de alta resolução, marca Topcon e modelo EM002B, operando a $200 \mathrm{kV}$ e com resolução ponto a ponto de $0,17 \mathrm{~nm}$. As áreas específicas dos materiais foram medidas em um aparelho volumétrico de adsorção de nitrogênio (BET), marca Counter e modelo SA 3100.

\section{RESULTADOS E DISCUSSÃO}

O crescimento de nanofibras de carbono sobre as fibras modificam a textura do feltro de grafite. O material precursor apresenta um ganho de $100 \%$ em peso, um crescimento da área específica de $1 \mathrm{~m}^{2} / \mathrm{g}$ à $100 \mathrm{~m}^{2} / \mathrm{g}$ e um aumento significativo da resistência mecânica, isto devido ao ancoramento entre fibras de grafite, ocasionado pelo entrelaçamento das nanofibras de carbono crescidas na superfície das fibras (Figura 3a). A existência de uma forte interação entre as fibras de grafite e as partículas de níquel pode explicar a boa dispersão do metal e, consecutivamente, a grande quantidade de nanofibras sintetizadas na superfície do suporte. É provável que, durante o tratamento térmico que precede a síntese de nanofibras, o níquel se converta em carbeto, o qual é mais reativo para a produção de nanofibras de carbono a partir da mistura etano e hidrogênio.

A Figura $3 b$ ilustra o crescimento de nanofibras de carbono em torno de uma parte descoberta de microfibra de grafite, porém isto não é freqüente, uma vez que as nanofibras recobrem totalmente a superfície do precursor. A Figura $3 \mathrm{c}$ mostra a alta densidade dos emaranhados de nanofibras de carbono sobre a superfície da fibra e apresenta ainda a ordem de grandeza das nanofibras. O diâmetro médio das nanofibras de carbono formadas é de aproximadamente $30 \mathrm{~nm}$, enquanto o seu comprimento é de algumas centenas de nanômetros. As nanofibras de carbono formadas apresentam uma excelente fixação ao suporte, não apresentando perdas de massa mesmo quando submetidas a alguns minutos sob ultra-som. Esta forte estabilidade deve-se à forte interação metal-suporte, bem como a um possível rearranjo do níquel durante os tratamentos térmicos posteriores à impregnação do metal. Este rearranjo produz policristais nos quais algumas faces decompõem o hidrocarboneto, enquanto outras propiciam o crescimento das nanofibras em diferentes direções. Uma parte das nanofibras produzidas penetra na rede de filamentos nanométricos, produzindo um entrelaçamento e apresentando pontos de colagem entre as nanofibras, que aumentam significativamente a resistência mecânica do material. As nanofibras formadas são constituídas de um empilhamento de planos cristalinos prismáticos de grafite ao longo do seu eixo. Estes planos apresentam sítios de adsorção mais reativos que os pertencentes ao plano basal, o que explica a forte interação metal-suporte.

A imagem de alta resolução feitas em MET das nanofibras de carbono impregnadas com $30 \%$ em peso de irídio é apresentada na Figura 3d. Esta figura mostra uma boa dispersão do metal sobre a superfície do suporte, dispersão similar àquela observada no catalisador comercial, o qual utiliza uma alumina como suporte ${ }^{11}$. O diâmetro médio das partículas após redução a $400{ }^{\circ} \mathrm{C}$ durante $2 \mathrm{~h}$ foi estimado em $2 \mathrm{~nm}$. Esta boa dispersão é atribuída à forte interação entre as partículas de irídio e os planos cristalinos expostos pelas nanofibras de carbono. Nesta figura pode-se também observar a estrutura das nanofibras de carbono na forma de "fishbone", ou seja, placas de grafite sobrepostas em ângulo e conectadas ao interior da fibra.

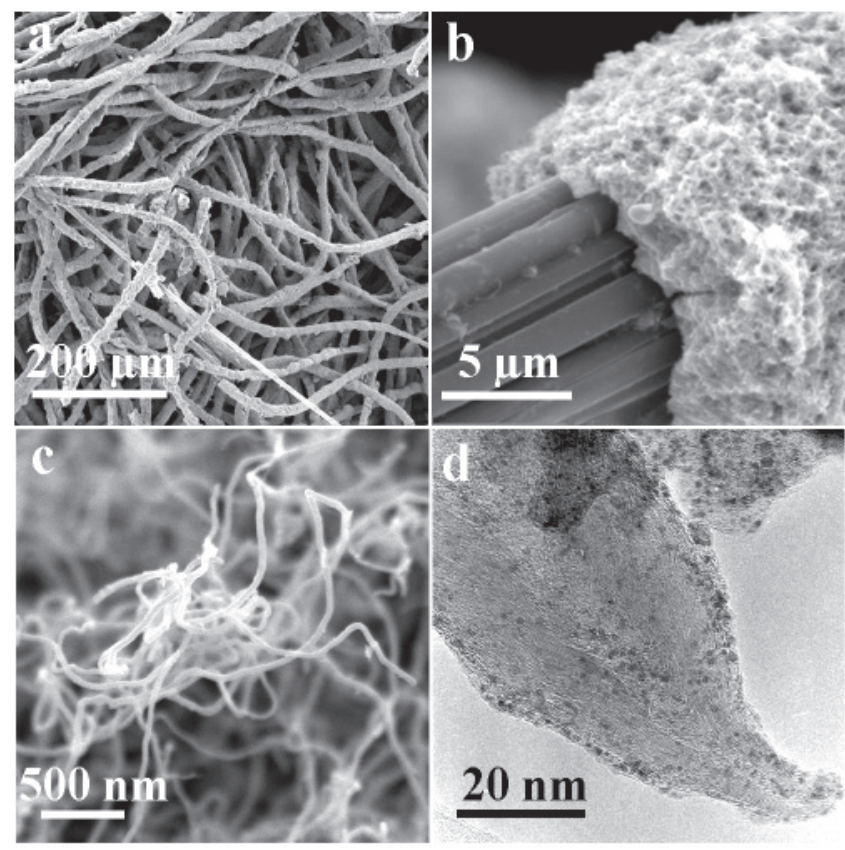

Figura 3. Imagens obtidas por microscopia eletrônica de varredura (a), (b) $e(c)$ e de transmissão $(d)$ de nanofibras de carbono crescidas sobre fibras de grafite comercial na forma de feltro

O desempenho do catalisador à base de nanofibras de carbono na reação de decomposição da hidrazina foi comparado ao do catalisador comercial (Figura 4). Os valores superiores de pressão de câmara apresentados pelos catalisadores à base de nanofibras de carbono podem ser atribuídos à excelente difusão de calor associada a maior condutividade térmica destes materiais, bem como à facilidade de difusão de massa devido a textura deste novo suporte, que facilita a acessibilidade do reativo e a evacuação dos produtos da reação.

$\mathrm{O}$ catalisador à base de nanofibras de carbono apresentou um bom desempenho na reação de decomposição da hidrazina e a sua performance aumenta com o desenrolar dos testes ${ }^{12}$. A morfologia do catalisador é conservada após os testes, não sendo observada a formação de finos. A boa estabilidade mecânica observada pode igualmente ser explicada pela ausência de porosidade neste material, pois o aumento drástico de pressão no interior dos poros pode causar fraturas no catalisador e conseqüentes perdas da fase ativa.

Os últimos testes efetuados com o catalisador comercial apresentaram instabilidade nos valores das pressões em função do tempo. Esta instabilidade pode estar associada à quebra do catalisador durante o ensaio, causada pela sobrepressão gerada no interior dos poros do catalisador. Após os testes, o catalisador comercial apresentou uma perda de massa na forma de finos da ordem de $20 \%$, enquanto o novo catalisador não apresentou perda de massa significativa.

Nas Figuras 5a e 5b pode-se observar grandes partículas de irídio formadas durante os testes de decomposição da hidrazina. A sinterização do metal está associada às altas temperaturas alcançadas na câmara de reação. A imagem de alta resolução (Figura 5c), obtida das partículas de irídio suportadas em nanofibras de carbono, após serem submetidas à reação de decomposição da hidrazina, mostra que uma parte do metal continua bem dispersa sobre a superfície do suporte, apresentando um tamanho médio de partícula da ordem de $3 \mathrm{~nm}$. Este fenômeno de movimentação de espécies sobre a superfície do catalisador pode ser minimizado através de um pré-tratamento do catalisador, visando eliminar o cloro residual proveniente do ácido precursor ${ }^{13}$. 

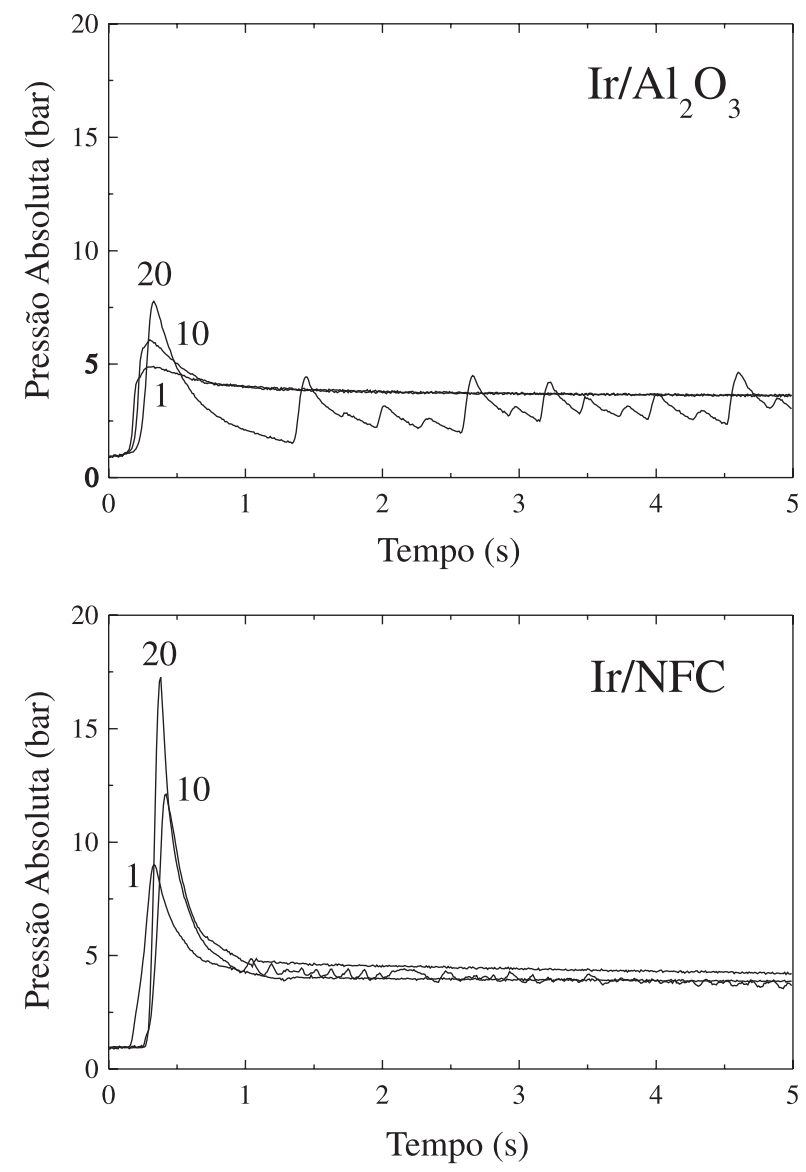

Figura 4. Gráfico comparativo dos desempenhos dos catalisadores comerciais $\left(\mathrm{Ir} / \mathrm{Al}_{2} \mathrm{O}_{3}\right)$ e Ir depositado em nanofibras de carbono (Ir/NFC), apresentando as curvas do $1^{\circ}$, do $10^{\circ}$ e do $20^{\circ}$ ensaio

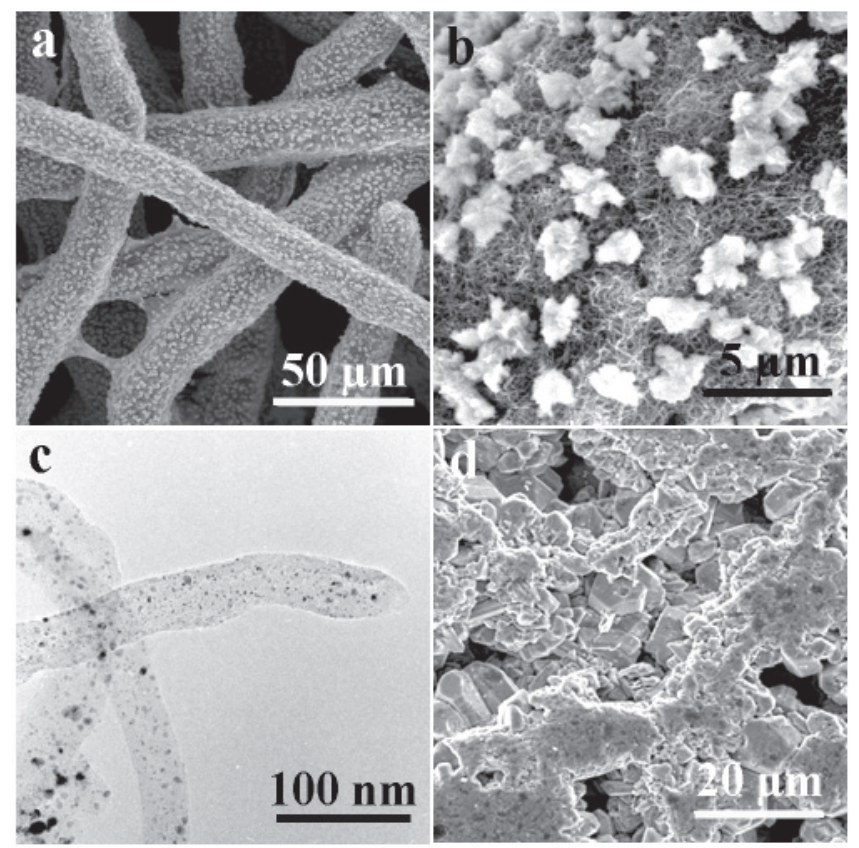

Figura 5. Imagens obtidas por microscopia eletrônica de varredura (a), (b) $e(d)$ e de transmissão (c) de partículas metálicas de irídio suportadas sobre nanofibras de carbono ( $a, b$ e c) e sobre alumina (d), após utilização na reação de decomposição da hidrazina
As imagens de MEV dos catalisadores, após 20 ensaios, mostram que a sinterização do irídio é mais acentuada no catalisador comercial (Figura 5d), onde pode-se observar que o metal praticamente se funde na superfície da alumina. Já no catalisador à base de nanofibras de carbono, o irídio migra formando grãos micrométricos, Figura $5 b$.

Os resultados obtidos por microscopia permitem uma explicação para o comportamento dos catalisadores durante os testes. No primeiro teste, para ambos os catalisadores, as partículas nanométricas de irídio permitem uma rápida decomposição da hidrazina injetada. No entanto, a elevada temperatura gerada durante a decomposição do propelente, induz a um princípio de sinterização nos dois catalisadores. Este fenômeno é menos acentuado no catalisador à base de nanofibras de carbono, pois neste material a alta condutividade térmica do suporte não favorece a formação de pontos superaquecidos na superfície e minimiza, desta forma, o fenômeno de sinterização.

O gráfico da Figura 4 resume uma seqüência de ensaios dos catalisadores na reação de decomposição da hidrazina em uma câmara confinada. Os resultados indicam que partículas maiores de irídio favorecem a decomposição do propelente, pois observou-se um aumento da sobrepressão da câmara de ensaios em função do número de testes. Em contrapartida, as pequenas partículas de irídio ( $<2 \mathrm{~nm}$ ) favorecem a diminuição do tempo de ignição. Assim, supõe-se que uma distribuição bimodal das partículas metálicas pode aumentar o desempenho do catalisador à base de nanofibras de carbono nesta reação.

\section{CONCLUSÕES}

Os resultados obtidos neste trabalho permitem concluir que é possível sintetizar novos materiais à base de nanofibras de carbono em uma forma macroscópica pré-definida. Esta síntese permite a obtenção de um material constituído exclusivamente de nanofibras de carbono sem a formação de impurezas indesejáveis, tais como carbono amorfo. O material assim obtido apresenta resistência mecânica e área específica $\left(100 \mathrm{~m}^{2} / \mathrm{g}\right)$ muito superiores às do feltro de carbono, preservando, contudo, a mesma densidade do material precursor. As nanofibras de carbono são formadas pelo empilhamento de planos cristalinos prismáticos ao longo do eixo da fibra, apresentando assim uma forte interação com a fase metálica depositada.

Os testes de decomposição catalítica da hidrazina mostraram que o catalisador à base de irídio suportado sobre este novo material apresenta um desempenho superior ao do catalisador comercial, quando submetidos a condições reacionais idênticas. A combinação dos resultados de decomposição catalítica e das imagens obtidas por microscopia eletrônica permite que se levante a hipótese de que a taxa de reação de decomposição da hidrazina é favorecida sobre grandes partículas (micrométricas) de irídio e que as pequenas partículas (nanométricas) determinam os valores do tempo de ignição da reação.

\section{AGRADECIMENTOS}

Os autores agradecem o apoio financeiro do Conselho Nacional de Desenvolvimento Científico e Tecnológico (CNPq), bem como a minuciosa revisão deste trabalho por parte do Dr. J. A. J. Rodrigues (LCP/INPE).

\section{REFERÊNCIAS}

1. Audrieth, L. F.; Ogg, B. A.; The Chemistry of Hydrazine, John Wiley \& Sons: New York, 1951.

2. Meetin, R. J.; Newton, N. C.; US pat. AD-786415 1974.

3. Schmidt, H. D.; Hydrazine and its Derivatives: Preapartion, Properties and Aplications, John Wiley: New York, 1984. 
4. Jun Imai, T.; Suzuki, T.; Kaneko, K.; Catal. Lett. 1993, 20, 133.

5. Dillon, A. C.; Jones, K. M.; Bekkedahl, T. A.; Kiang, C. H.; Bethune, D. S.; Heben, M. J.; Nature 1997, 386, 377.

6. Krätschmer, W.; Lamb, L. D.; Fostiropoulos, K.; Huffman, D. R.; Nature 1990, 347, 354.

7. Thess, A.; Lee, R.; Nikolaev, P.; Dai, H.; Petit, P.; Robert, J.; Xu, C.; Young Hee Lee, A. G.; Seong Gon Kim, A. G.; Rinzler, A. G.; Colbert, D. T.; Scuseria, G. E.; Tomanek, D.; Fischer, J. E.; Smalley, R. E.; Science 1996, 273, 483.

8. Rodriguez, N. M.; J. Mater. Res. 1993, 8, 3233.
9. Ajayan, P. M.; Zhou, O. Z.; Carbon Nanotubes: Synthesis, Structure, Properties and Applications, Springer Verlag: Heidelberg, 2001.

10. Pham-Huu, C.; Vieira, R.; Ledoux, M. J.; Charbonnière, L.; Ziessel, R.; French pat. 01151782001.

11. Cunha, D. S.; Tese de Doutorado, Faculdade de Engenharia Química de Lorena, Brasil, 1995.

12. Vieira, R.; Pham-Huu, C.; Keller, N.; Ledoux, M. J.; Chem. Commun. 2002, 954.

13. Balcon, S.; Mary, S.; Keppenstein, C.; Gengembre, E.; Appl. Catal., A 2000, $196,179$. 